\title{
DETERMINAÇÃO DE ALA-U EM TRABALHADORES DE AUTO ELÉTRICAS E FUNILARIAS EXPOSTOS OCUPACIONALMENTE AO CHUMBO NA CIDADE DE PRESIDENTE PRUDENTE, SP
}

\author{
Camila Baldi Rodrigues, Izabela Esteves Domingues, Angélica Augusta Grigoli Dominato \\ Universidade do Oeste Paulista, Curso de Biomedicina, Presidente Prudente, São Paulo. e-mail:angelica@unoeste.br
}

\begin{abstract}
RESUMO
A monitorização biológica é utilizada para acompanhar e minimizar a ocorrência de intoxicação de trabalhadores em ambiente ocupacional. Realizar a monitorização biológica por meio do ácido delta aminolevulínico - urinário (ALA-U) de trabalhadores expostos ao chumbo foi objetivo desta pesquisa. O método de espectrofotometria na região do visível foi utilizado para determinação de ALA-U. Os valores de ALA-U estiveram entre 0,5 a $5,5 \mathrm{mg} / \mathrm{L}$, dentre os participantes $65 \%$ relataram o uso de EPI e 75\% de EPC. A saúde do trabalhador vincula-se às práticas de higiene e uso de equipamentos de proteção assim como a consulta anual ao médico do trabalho e realização de exames periódicos. A realização de exames laboratoriais periódicos deve ser estimulada pelos empregadores para a manutenção da saúde ocupacional de seus empregados.

Palavras-chave: chumbo, intoxicação ocupacional, ALA-U, funilarias, auto elétricas.

\section{DETERMINATION OF ALA-U IN CAR ELECTRICAL REPAIR SHOPS AND BODY SHOPS WORKERS OCCUPATIONALLY EXPOSED TO LEAD FROM PRESIDENTE PRUDENTE, SP.}

\begin{abstract}
Biological monitoring is used to monitor and minimize the occurrence of poisoning by workers in the occupational environment. Perform biological monitoring through the delta aminolevulinic acid - urine (ALA-U) of workers exposed to lead was the objective of this research. The spectrophotometric method in the visible region was used for determination of ALA-U. The ALA-U values were from 0.5 to $5.5 \mathrm{mg} / \mathrm{L}$, among the participants $65 \%$ reported the use of PPE and $75 \%$ $\mathrm{CPE}$. The worker's health is linked to the hygiene practices and use of protective equipment as well as the annual consultation of the doctor's work and performing periodic examinations. Encourage workers exposed to lead in realize laboratory tests should be stimulated by employers and consequently the maintenance of occupational health.
\end{abstract}

Keywords: lead, occupational poisoning, ALA-U, body shops, car electrical repair shops. 


\section{INTRODUÇÃO}

A intoxicação por chumbo é uma das mais importantes doenças ambientais e ocupacionais causada pelos elevados níveis do metal no ambiente, ocorrendo especialmente em áreas altamente industrializadas, provocando frequentemente disfunções hematológicas, endócrinas, gastrointestinais e neurológicas (GODINHO et al., 2001).

O chumbo expõe o organismo humano por três vias principais: respiratória, cutânea e oral, sendo a respiratória a via mais importante. Neste caso, ocorre a absorção de $20-40 \%$ do chumbo inalado na forma de vapores (compostos orgânicos), fumos (óxido de chumbo) ou poeiras (AZEVEDO; CHASIN, 2003; LEAL, 2009).

Após a absorção de altas quantidades de chumbo, e sua distribuição, o metal atinge o sistema nervoso, a medula óssea e os rins, que são considerados sítios críticos na exposição ao chumbo. A interação deste metal na medula óssea causa distúrbios da síntese do grupamento heme (hemoglobina), que, na toxicologia ocupacional é considerado efeito tóxico crítico (MOREIRA; MOREIRA, 2004).

Outros efeitos são observados na intoxicação pelo chumbo, com mudanças nos processos bioquímicos, que podem ser divididos em três grupos. O primeiro está relacionado à sua ligação com vários doadores de elétrons, particularmente a grupos sulfídricos, que induzem às alterações nas proteínas, principalmente na ação enzimática. O segundo é resultante da semelhança biofísica com o cálcio, o que permite o acesso aos locais críticos da célula, como as mitocôndrias. Por fim, o chumbo parece afetar os ácidos nucléicos e induzir aberrações cromossômicas por meio de mecanismos ainda não definidos. O chumbo pode, também, causar danos à saúde, mesmo dentro dos limites considerados legais, desencadeando comprometimento da memória, do humor, e da coordenação motora em trabalhadores expostos ao chumbo com níveis aceitáveis e frequentes (MINOZZO et al., 2008).

A ocupacional é a forma principal de contato com o chumbo em níveis perigosos, por isso torna-se importante o acompanhamento médico-laboratorial dos níveis de chumbo no organismo dos trabalhadores expostos. Para tanto estão previstos, na legislação brasileira, como parâmetros para controle biológico da exposição ocupacional os seguintes indicadores biológicos: $\mathrm{Pb}-\mathrm{S}, \mathrm{ALA}-\mathrm{U}$ e ZPP-S. Essa norma também estabelece como parâmetros o Valor de Referência de Normalidade (VR) e o Índice Biológico Máximo Permitido (IBMP), que, para o Pb-S de $40 \mu \mathrm{g} / \mathrm{dL}$ e de $60 \mu \mathrm{g} / \mathrm{dL}$, respectivamente (ARAÚJO; PIVETA; MOREIRA, 1999).

O objetivo deste trabalho foi monitorizar o ALA-U (ácido delta aminolevulínico urinário) em trabalhadores de funilaria e auto elétricas expostos ao chumbo e associar os resultados laboratoriais às práticas de higiene das mãos, uso de equipamentos de proteção individual (EPI), equipamentos de proteção coletiva (EPC) e hábitos de consumo de cigarro.

\section{METODOLOGIA}

O projeto foi aprovado no Sistema Gestor de Pesquisa com o protocolo 2369, assim como na Plataforma Brasil com o número CAAE40073214.2.0000.5515.

O público alvo foi composto de 20 funcionários de funilarias e auto elétrica, sendo 18 funcionários de quatro funilarias e 2 funcionários de uma auto elétrica, localizadas na cidade de Presidente Prudente - SP, escolhidas de forma aleatória. Inicialmente, os trabalhadores foram convidados a participar da pesquisa, por meio de carta convite, e ao aceitarem assinaram o termo de consentimento livre e esclarecido. Os critérios de inclusão foram a obrigatoriedade de exposição ocupacional ao chumbo: todos os trabalhadores estavam expostos há mais de um ano ao metal e realizaram a jornada semanal de trabalho completa para a coleta das amostras, além de terem idade superior aos 18 anos. A intensidade de exposição com possíveis níveis de intoxicação está vinculado ao tempo de exposição, hábitos de higiene, uso de EPI e EPC. Proporcionalmente, as auto elétricas apresentam maiores riscos de exposição, devido ao 
manuseio de baterias, compostas por placas de chumbo. Enquanto que, nas funilarias o risco de exposição ao chumbo está no manuseio das tintas automotivas, sendo então problemas pontuais de exposição.

Todos os voluntários eram masculino e maiores de 18 anos. Foi aplicado um questionário contendo perguntas pertinentes às condições de trabalho e possíveis exposições do trabalhador fora da rotina diária de trabalho. As amostras de urina para determinação de ALA-U foram coletadas no último dia útil da semana do expediente de trabalho, com o procedimento de coleta para exame de urina, isolada, tipo I.

Após a coleta de urina dos voluntários, as mesmas foram encaminhadas ao laboratório de Toxicologia e mantidas em freezer a $-20^{\circ} \mathrm{C}$ até o momento da realização da análise. As dosagens de ALA-U nas urinas coletadas foram realizadas no mesmo dia e em triplicata. No dia da coleta das amostras de urina foi aplicado um questionário aos voluntários contendo informações sobre tempo de exposição, uso de EPI e EPC, hábitos de higiene pessoal, consumo de cigarro de tabaco e de bebidas como chá e café.

Os trabalhadores foram orientados, no momento da aceitação de participação do projeto, que a urina deveria permanecer na bexiga por período mínimo de três horas. A determinação de ácido delta aminolevulínico urinário - ALA-U, foi realizado em pH tamponado, e o ALA foi extraído da urina pelo acetato de etila e quantificado após a reação com o reativo de Ehrlich modificado e as absorbâncias foram obtidas em $553 \mathrm{~nm}$, em espectrofotômetro no visível (MORAES; SZNELWAR; FERNÍCOLA, 1991; MOREAU; SIQUEIRA, 2008).

Os valores aobtidos foram comparados com os resultados elencados na Tabela 1.

Tabela 1. Valores de referência para ALA-U.

\begin{tabular}{cc}
\hline Classificação & Valor de referência $(\mathrm{mg} / \mathrm{L})$ \\
\hline Normal & $<6$ \\
Aceitável & 6 a 20 \\
Excessivo & 20 a 40 \\
Perigoso & $>40$
\end{tabular}

Fonte: MORAES; SZNELWAR; FERNÍCOLA, 1991.

A amostra representativa de funcionários de funilaria e auto elétrica seriam de 30 voluntários, entretanto a coleta ficou prejudicada em sua totalidade, pois os voluntários relataram que esqueciam de coletar a primeira amostra do dia ou não concordaram em participar da projeto.

\section{RESULTADOS}

Os resultados obtidos das dosagens de ALA-U estão elencados na Tabela 2, e verificouse que todos os participantes apresentam valores inferiores a $6 \mathrm{mg} / \mathrm{L}$ (variam entre 0,5 a 5,5 $\mathrm{mg} / \mathrm{L}$ ), mesmo para aqueles que estão expostos há mais tempo. Dentre os valores obtidos, $75 \%$ apresentaram ALA-U entre 0,5 a 3,0 mg/L, e $15 \%$ entre 5,1 a 5,5 mg/L. Neste caso, eram 3 funcionários, com concentrações de $5,3 \mathrm{mg} / \mathrm{L}, 5,4 \mathrm{mg} / \mathrm{L} \mathrm{e} \mathrm{5,5} \mathrm{mg/L.}$ 
Tabela 2: Resultados das determinações de ALA-U de cada participante da pesquisa em mg/L

\begin{tabular}{cc}
\hline $\begin{array}{c}\text { ALA-U } \\
(\mathrm{mg} / \mathrm{L})\end{array}$ & $\begin{array}{c}\text { Voluntários } \\
(\%)\end{array}$ \\
\hline $0,5-1,0$ & 35 \\
$1,1-2,0$ & 25 \\
$2,1-3,0$ & 15 \\
$3,1-4,0$ & 5 \\
$4,1-5,0$ & 5 \\
$5,1-$ & \\
5,5 & 15 \\
\hline
\end{tabular}

A Tabela 3 apresenta os valores relativos da exposição ocupacional ao chumbo em relação ao tempo de exposição. Dentre os participantes $15 \%$ não responderam ao questionamento do tempo de exposição. Os valores observados demonstrou que $35 \%$ trabalhava entre 2 a 5 anos expostos ao chumbo e $25 \%$ dos voluntários trabalharam expostos ao chumbo entre 21 a 35 anos. Mesmo com exposição ao longo prazo, constatou-se valores seguros de ALA-U no momento da coleta de amostras.

Tabela 3: Classificação dos voluntários expostos ocupacionalmente ao chumbo em relação ao tempo de exposição em anos.

\begin{tabular}{cc}
\hline $\begin{array}{c}\text { Tempo de exposição } \\
\text { (anos) }\end{array}$ & Valor relativo (\%) \\
\hline $2-5$ & 35 \\
$6-10$ & 5 \\
$11-15$ & 5 \\
$16-20$ & 15 \\
$21-25$ & 5 \\
$26-30$ & 10 \\
$31-35$ & 10 \\
\hline
\end{tabular}

Quanto ao uso de EPI e EPC, foi possível observar mais adesão ao uso de EPC em relação ao EPI, essa adesão pode ser observada na Figura 1. A justificativa para tal diferença, de acordo com os participantes, é que o uso de EPI atrapalha o desenvolvimento do trabalho, não se acostumaram ao uso do mesmo ou por sentirem-se incomodados ao realizar o trabalho equipados com os referidos equipamentos. 


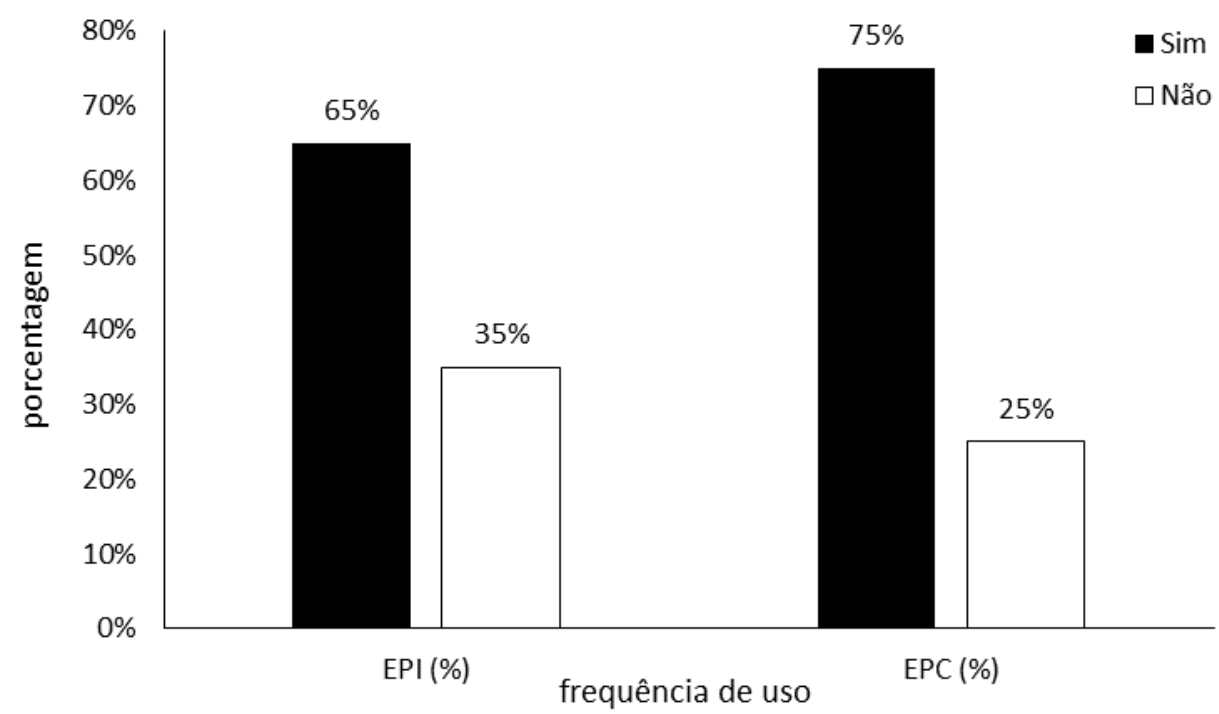

Figura 1. Frequência de uso de EPI e EPC relatado pelos voluntários da pesquisa.

O hábito do consumo de bebidas no ambiente laboral, como chá e café, esteve presente em $80 \%$ dos entrevistados, sendo que $10 \%$ relataram consumir as duas bebidas, e o hábito de fumar esteve presente em $5 \%$ dos entrevistados. As bebidas relatadas e o cigarro podem conter chumbo, somado ao consumo sem a prática de higiene das mãos contribuem para o aumento do risco de intoxicação no ambiente de trabalho.

A prática de higienização das mãos antes de utilizar o sanitário e consumir alimentos e bebidas foi um parâmetro analisado, onde os trabalhadores relataram realizar com frequência. Os cuidados relacionados ao uso de EPI e EPC, além das práticas de higiene pessoal, contribuíram fortemente para a determinação da saúde ocupacional dos trabalhadores expostos ao chumbo que participaram desta pesquisa.

O participante cuja concentração estava em $5,3 \mathrm{mg} / \mathrm{L}$ relatou trabalhar há 35 anos com exposição ao chumbo e que já realizou tratamento de desintoxicação do referido metal. Os voluntários que apresentaram resultados de ALA-U de 5,4 mg/L e 5,5 mg/L disseram que trabalham no setor de funilaria há 5 anos e que utilizavam os EPI e EPC durante a jornada de trabalho. Portanto, não pode ser afirmado que os valores das concentrações de ALA-U estiveram menores para aqueles trabalhadores que utilizavam EPI e EPC durante a jornada de trabalho.

\section{DISCUSSÃO}

Segundo Gomes (2013) e Clark, Oliveira e Clark (2010) relataram que os parâmetros mais importantes e necessários para uma avaliação rígida de salubridade estão nas instalações do ambiente de trabalho, assim como uso de EPI e EPC, tempo de exposição, hábitos alimentares e de higiene pessoal e atenção à saúde.

Em relação aos resultados de ALA-U, segundo o que preconiza o Ministério da Saúde (2006), a concentração chumbo no sangue, que indica início de uma possível intoxicação ocupacional é partir de $40 \mu \mathrm{g} / \mathrm{dL}$. Segundo a Portaria GM/MS/777, de 28 de abril de 2004, toda intoxicação ocupacional pelo chumbo deve ser notificada compulsoriamente, e em seguida comunicada à Previdência Social, por caracterizar acidente de trabalho (BRASIL, 2006).

Em um estudo realizado por Minozzo e colaboradores (2009) em 53 trabalhadores de indústria e reciclagem de baterias automotivas, demonstrou que $20,8 \%$ dos trabalhadores apresentaram níveis sanguíneos de chumbo dentro da normalidade, 35,8\% dos trabalhadores apresentaram resultados da plumbemia entre os valores de referência normais e o Índice Biológico Máximo Permitido (IBMP) e 43,4 \% apresentaram níveis superiores ao IBPM. 
Na pesquisa não foi realizada a dosagem de chumbo sanguíneo, entretanto as análises de ALA-U obtidas pelas amostras de urina estavam dentro dos parâmetros normais, como demonstrado nos resultados obtidos.

Prista, Uva e Aguiar (2003) confirmaram a validação do ALA-U como indicador de efeito da exposição ocupacional ao chumbo. Outro parâmetro comparado foi a dosagem de ALA-U em amostras de urina de $24 \mathrm{~h}$ e amostras de urina isolada e determinaram que não houve diferença significativa entre os resultados obtidos.

\section{CONCLUSÃO}

A concentração de ALA-U dos participantes da pesquisa estiveram dentro dos valores de referência. Este pode ser o resultados da manutenção de um ambiente de trabalho salubre em conjunto com a higiene pessoal. Estes aspectos contribuem para que o trabalhador se mantenha saudável e mais disposto para a realização da jornada diária, reduzindo drasticamente a possibilidade de ocorrência de prejuízos à saúde ao longo prazo. Entretanto é importante a realização de exames periódicos específicos para a exposição ao chumbo, que contribui para a manutenção da saúde do trabalhador, assim como o empregador deve estimular a prática de consultas periódicas ao médico do trabalho.

\section{REFERÊNCIAS}

Araújo UC, Piveta FR, Moreira JC. Avaliação da exposição ocupacional ao chumbo: proposta de uma estratégia de monitoramento para prevenção dos efeitos clínicos e subclínicos. Cadernos de saúde pública. 1999; 15(1): 123-131. DOI: http://dx.doi.org/10.1590/S0102-311X1999000100013.

Azevedo FA, Chasin AAM. Metais: gerenciamento da toxicidade. São Paulo, Rio de Janeiro, Belo Horizonte: Atheneu; 2003.

Brasil, Ministério da Saúde. Atenção á Saúde dos Trabalhadores Expostos ao Chumbo Metálico. Brasília: Editora MS, 2006.

Clark LGO, Oliveira HG, Clark OAC. A exposição ocupacional ao chumbo e os riscos à saúde do trabalhador. Jornal Brasileiro de Economia da Saúde. 2010; 2(1): 8-14. Disponíel em http://www.evidencias.com.br/pdf/publicacoes/ff4ea810415e1e88d6e1b2f358d65cae.pdf.

Godinho AF, Vassilief I, Almeida AA, Silva AG, Aguiar DC. Intoxicação por chumbo em bebês lactentes em três casos de exposição maternal. Salusvita. 2001; 20(3): 11-18.

Gomes SF. A importância e aplicabilidade da Odontologia do trabalho na exposição ambiental ao chumbo. Revista Brasileira de Odontologia. 2013; 70(1): 85-88. Disponível em http://revista.abori.org.br/index.php/rbo/article/viewFile/417/368.

Leal PC. Exposição ocupacional ao chumbo e seus compostos. Universidade Federal de Ouro Preto. 2009.

Minozzo R, Minozzo EL, Deimling LI, Mello RS. Plumbemia em trabalhadores da indústria de reciclagem de baterias automotivas da grande Porto Alegre, RS. Jornal Brasileiro de Patologia e Medicina Laboratorial. 2008; 44(6): 407-412. DOI: http://dx.doi.org/10.1590/S167624442008000600003. 
Minozzo R, Wagner SC, Santos CH, Deimling LI, Mello RS. Prevalência de anemia em trabalhadores expostos ocupacionalmente ao chumbo. Revista Brasileira de Hematologia e Hemoterapia. 2009; 31(2). DOI: http://dx.doi.org/10.1590/S1516-84842009005000018.

Moraes ECF, Sznelwar RB, Fernícola NAGG. Manual de toxicologia analítica. Editora Roca, 1991. p. 47-49.

Moreau RLM, Siqueira M E P B. Toxicologia analítica. Rio de Janeiro: Guanabara Koogan, 2008.

Moreira FR, Moreira, JC. A importância da análise de especiação do chumbo em plasma para a avaliação dos riscos à saúde. Química Nova. 2004; 27(2): 251-260. DOI: http://dx.doi.org/10.1590/S0100-40422004000200015

Prista J, Uva AS, Aguiar P. Exposição profissional ao chumbo: utilização do doseamento do ácido $\delta$ aminolevulínico urinário na vigilância médica dos trabalhadores. Revista Portuquesa de Saúde Pública. 2003; 21(1): 17-22. Disponível em: https://www.ensp.unl.pt/dispositivos-deapoio/cdi/cdi/sector-de-publicacoes/revista/2000-2008/pdfs/1-02-2003.pdf 